\title{
Design of Double-drive Mechanism for Energy Saving Lifting Permanent Magnet
}

\author{
Ding Ning*, Liu Chao, Duan Jingsong, and Jiang Shuna \\ Mechanical and Vehicle Engineering College, Changchun University, Changchun 130022, China
}

\begin{abstract}
On account of great power consume of lifting electromagnet, energy-saving lifting permanent magnet has got more attention. But in the exiting design of lifting magnet, most can only lift the workpiece placed horizontally, and can't lift the workpiece placed inclined. Aiming at this defect, the authors design the double-drive mechanism. In this design, two sets of tumble, cylindrical gear mechanism and ratchet pawl mechanism were used to ensure that at least one set of mechanisms can work properly in the inclined state. To enable the two sets of mechanisms to drive the rotating magnetic system in the same direction, reversing gear was adopted.
\end{abstract}

\section{Introduction}

Lifting electromagnet is a kind of magnetic transport equipment which realizes loading and unloading by the current control. It has been widely used in iron and steel smelting, metal mine, shipbuilding, port transportation, rail logistics and other industries. Because of its magnetic field comes from the magnetization of soft magnet by electric coil, it needs to consume huge electric energy when it works. According to statistics, a 20 kilowatt lifting electromagnet works 12 hours every day, 300 days every year, power consumption up to 72000 kilowatt-hour. In Anshan Iron and Steel Group Corporation, there are 300 lifting electromagnet working every day. If from national point of view, every year the electricity consumption of electromagnet is inestimable! Hence, if it can be replaced, it will save energy and reduce emissions.

Lifting permanent magnet is a new type of magnetic transport device, the permanent magnets is used as its magnetic source, the principle of magnetic field superposition is used to realize the superposition and cancellation of magnetic field between fixed magnetic system and rotating magnetic system. Due to the mechanism structure is used as drive system, its power consumption is zero in full work cycle. Compared with huge power consumption of lifting electromagnet, the permanent magnet crane technology is a subversive technology. It fits the development strategy of energy conservation and emission reduction.

Since the 1960s, scholars from Kepler University in France put forwards the idea of developing lifting permanent magnet, scholars in home and abroad have done a lot of research in this field. In 1992, M.X. Wang, N. Ding, C.C. Gao and L.G. Ding design a magnetic circuit with rotating magnetic system and fixed magnetic system [1]. The magnetic field intensity of magnetic pole surface of this design is less than 600 e when unloading, that is, less than the magnetic intensity that attracts iron fillings. This design circuit is also the most commonly used design ideas of lifting permanent magnet. In 2004, Ding Ning design the lifting permanent magnet for iron and steel scrap [2-6]. In 2013, G.F. Yang, T.G. Chen utilize piston rod, gear and gear rack to drive the rotating magnetic system [7]. After many years development, the lifting permanent magnet is still imperfect, which is that when the ferromagnetic workpiece is placed at a certain angle to horizontal ground, the lifting permanent magnet couldn't work normally. In view of this shortcoming, this paper designs a kind of double-drive mechanism, which makes the lifting permanent magnet able to lift the inclined workpiece.

\section{Working principle of magnetic circuit}

As a magnetic transport equipment, lifting permanent magnet has two basic tasks: first, able to hold workpiece reliably; second, unload workpiece fast and convent. For lifting permanent magnet, it is more difficult to unload workpiece than hold workpiece. So how to unload workpiece is the most critical technical challenge.

According to the principle of magnetic field superposition, we have designed a magnetic circuit which can realize load and unload fast and convent. The design of the magnetic circuit is shown in Fig 1. The whole magnetic circuit is composed of magnetizer, fixed magnetic system and rotating magnetic system. When the pole $\mathrm{N}$ of rotating magnetic system goes up, the magnetic field of rotating magnetic system superimposed on the fixed magnetic system, and lifting permanent magnet loads workpiece, as shown in Fig. 2. (a). When the pole N of rotating magnetic system goes down, the magnetic field of rotating and fixed magnetic system cancel each other

${ }^{*}$ Corresponding author: $\underline{\operatorname{dn} 33 \mathrm{cn} @ 163 . c o m}$ 
out, and lifting permanent magnet unloads workpiece, as shown in Fig. 2. (b).

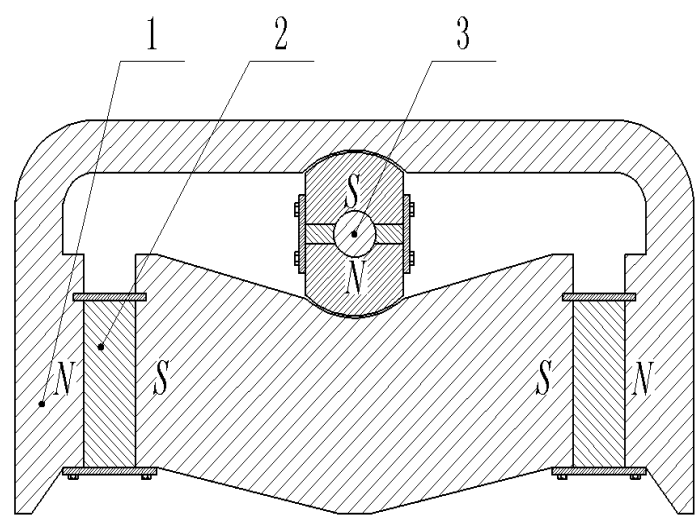

1-magnetizer, 2-fixed magnetic system, 3- rotating magnetic system

Fig. 1. Diagram of magnetic circuit

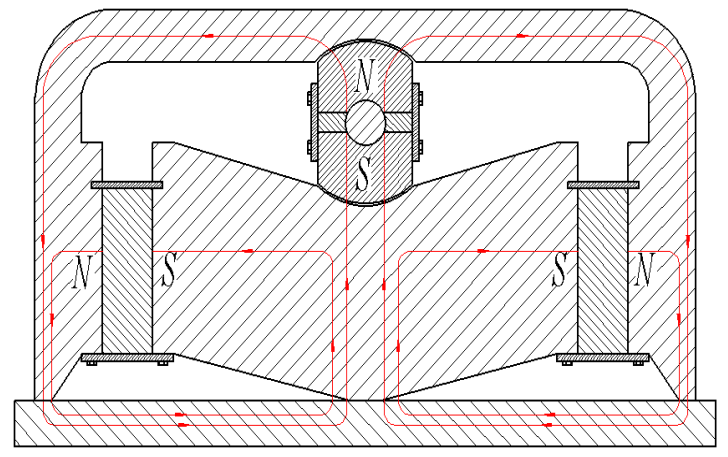

(a) Loading workpiece magnetic circuit

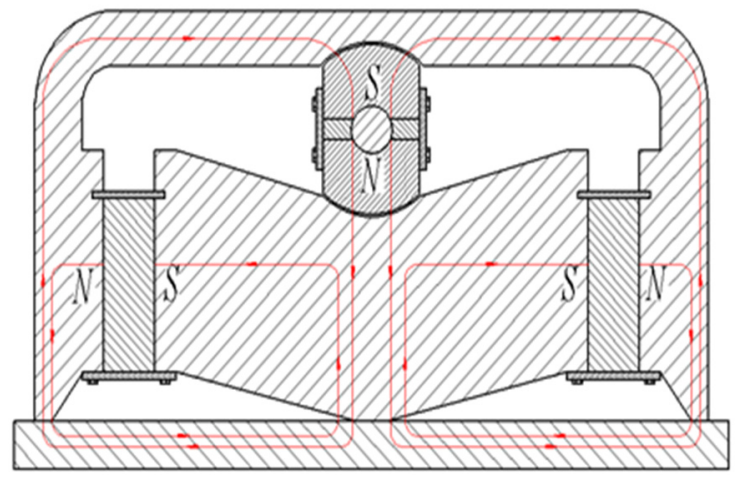

(b) Unloading workpiece magnetic circuit

Fig. 2. Magnetic circuit working process

\section{Design of double-drive mechanism}

The drive system is the essential part of lifting permanent magnet. If it couldn't drive the magnetic system to rotate, most weight lifting is meaningless. The design of driving system need to meet the following requirements: fast and convent unloading and enables it to work properly in an inclined state. According to the requirement, we designed a set of double-drive mechanism. It is shown in Fig. 3. (a) and (b).
The double-drive mechanism contains inside big gear, inside small gear, hollow shaft, outside small gear, outside big gear, reversing gear, shaft, left tumbler, right tumbler, left small chain, right small chain, lifting chain, left ratchet, right ratchet and pawl. The inside big gear and the left ratchet are fixed with hollow shaft through the key and the left tumbler fits on the hollow shaft. The outside big gear and right ratchet are fixed with the shaft through the key and the right tumbler fits on the shaft. The inside big gear engage with inside small gear, the outside big gear, the outside small gear and the reversing gear engaged with each other. The inside small gear and the outside small gear are fixed with the magnetic poles half shaft through the key. The pawl is installed in the right and left tumbler and matched with the left and right ratchet. The hollow shaft is fitted on the shaft. The left and right small chain linked with the left and right tumbler. The lifting chain, left and right small chain linked with the lifting hook.

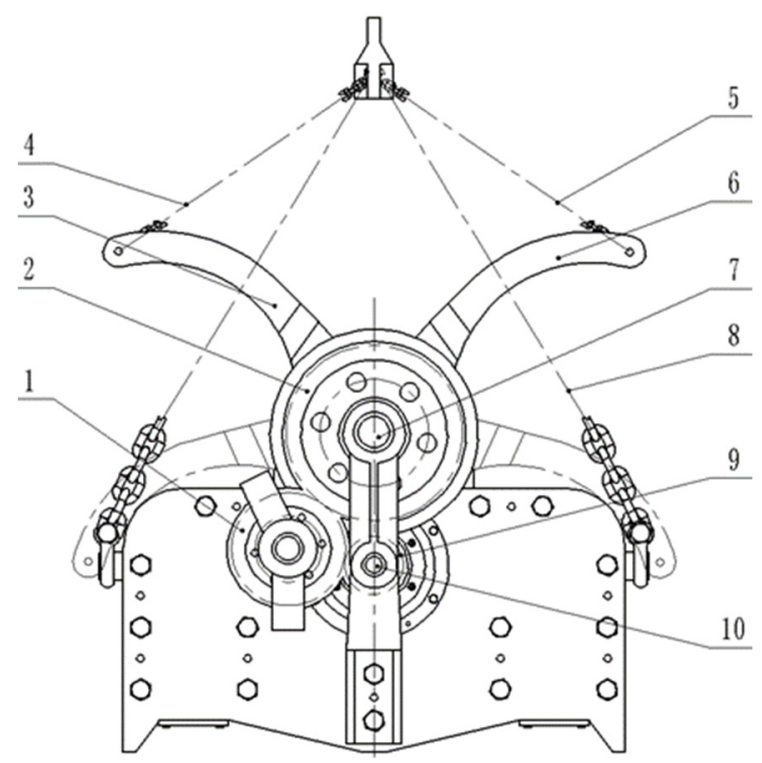

(a) Lifting permanent magnet front view

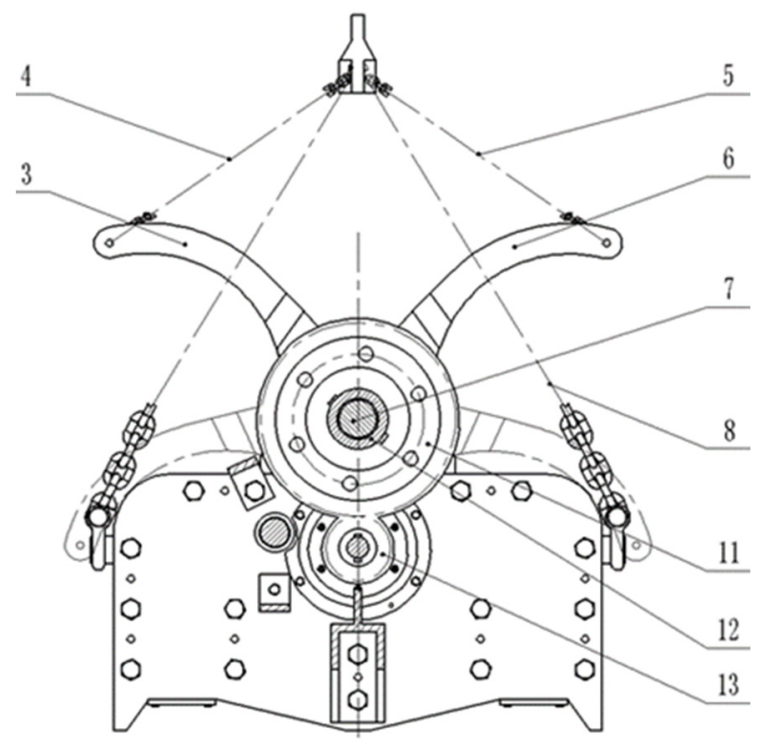

(b) Lifting permanent magnet front view (remove outside gear and reversing gear) 
1-reversing gear, 2-outside big gear, 3-left tumbler, 4-left small chain, 5-right small chain, 6-right tumbler, 7-shaft, 8-lift chain, 9-outside small gear, 10- magnetic poles half shaft, 11-inside big gear, 12-hollow shaft, 13-inside small gear

Fig. 3. Lifting permanent magnet double-drive mechanism

\subsection{Process of lifting the flat workpiece}

When the lifting permanent magnet is placed on the workpiece, the small chain linked to lifting hook is tightened and the left and right tumbler are lifted. The pawls which installed in the left and right tumbler push the left and right ratchet rotation. The inside big gear rotates clockwise and push the inside small gear counterclockwise. The outside big gear rotates counterclockwise and push the reversing gear clockwise and push the outside small gear counterclockwise. Under the joint action of the inside and outside small gear, the rotating magnetic system rotates 180 degrees to realize load workpiece.

\subsection{Process of lifting the inclined workpiece}

When the lifting permanent magnet is placed on the inclined workpiece, only one of the small chain can tightened, the other is slacked. In the actual process, we cannot determine which small chain is slacked and which one is tightened. So, we designed the double-drive mechanism. When one of the tumble is lifted, it still drives the rotating magnetic system rotating 180 degrees.

Take the example of a workpiece inclined to the left, as shown in Fig. 4. When the lifting permanent magnet is placed on the workpiece, the lifting hook is lifted, the left small chain is tightened first, and the right is slacked. The pawls which installed in the left tumbler push the left ratchet rotation. The inside big gear rotates clockwise and push the inside small gear counterclockwise, the rotating magnetic system rotates 180 degrees to realize load workpiece.

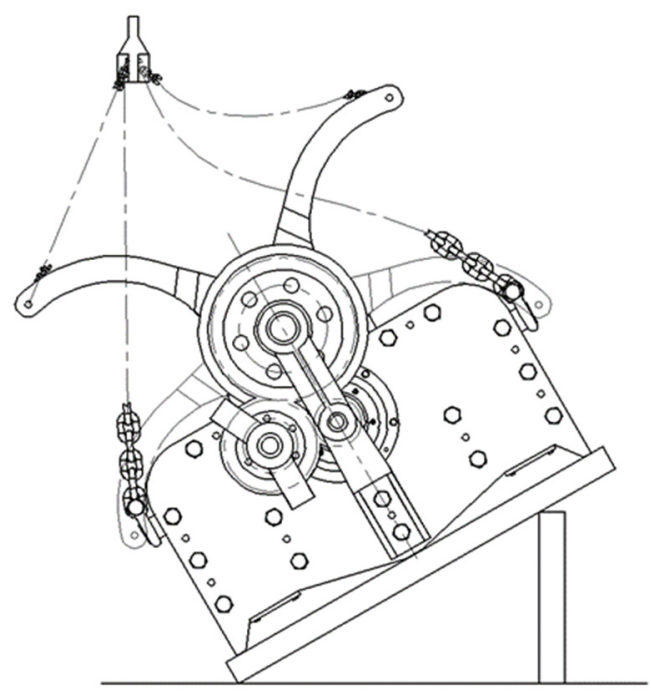

Fig. 4. Schematic diagram of lifting workpiece inclined to the left

\subsection{Process of unload workpiece}

When the lifting permanent magnet lifts the workpiece into the place for unloading, the lift hook down, chain is slaked. Under the action of gravity, the tumblers reach its lowest point. The pawls slides to the next tooth of the ratchet. After that, the lift hook lift, repeat the process of lifting to make the rotating magnetic system rotate 180 degrees again, its magnetic field cancel out with fixed magnetic system, unload workpiece.

\section{Conclusions}

By now, the author have designed and manufactured the double-drive lifting permanent magnet. The design is proven to be feasible. Regardless of the inclined state of the workpiece, double-drive mechanical structure can make the lifting permanent magnet work properly, which broadens its scope of use.

The authors would like to acknowledge funding support from Jilin Province Industrial Technology Research and Development Project (Grant No. 2018C043-2).

\section{References}

1. X.M. Wang, N. Ding, C.C. Gao, China Patent No. 92240596.4, (1994)

2. N. Ding, China Patent No. 02126633.6, (2004)

3. N. Ding, D.T. Zhang, Y.M. Song, Adv. Mater. Res. 765-767, 125-128(2013)

4. N. Ding, D.T. Zhang, Z.Z. Wang, Appl. Mech. Mater. 670-671, 950-954 (2014)

5. N. Ding, D.T. Zhang, Adv. Mater. Res. 569, 391-394 (2012)

6. N. Ding, D.T. Zhang, Y.M. Song, J. Shi, L.G. Ding, Adv. Mater. Res. 662, 653-656 (2013)

7. G.F. Yang, T.G. Chen, China Patent No. 201320718334.3, (2014) 